PREPARED FOR THE U.S. DEPARTMENT OF ENERGY, UNDER CONTRACT DE-AC02-76CH03073

PPPL-3793

PPPL-3793

UC-70

Ion Acceleration in Plasmas Emerging from a Helicon-heated Magnetic-mirror Device

by

S.A. Cohen, N.S. Siefert, S. Stange, R.F. Boivin,

E.E. Scime, and F.M. Levinton

March 2003

NM|

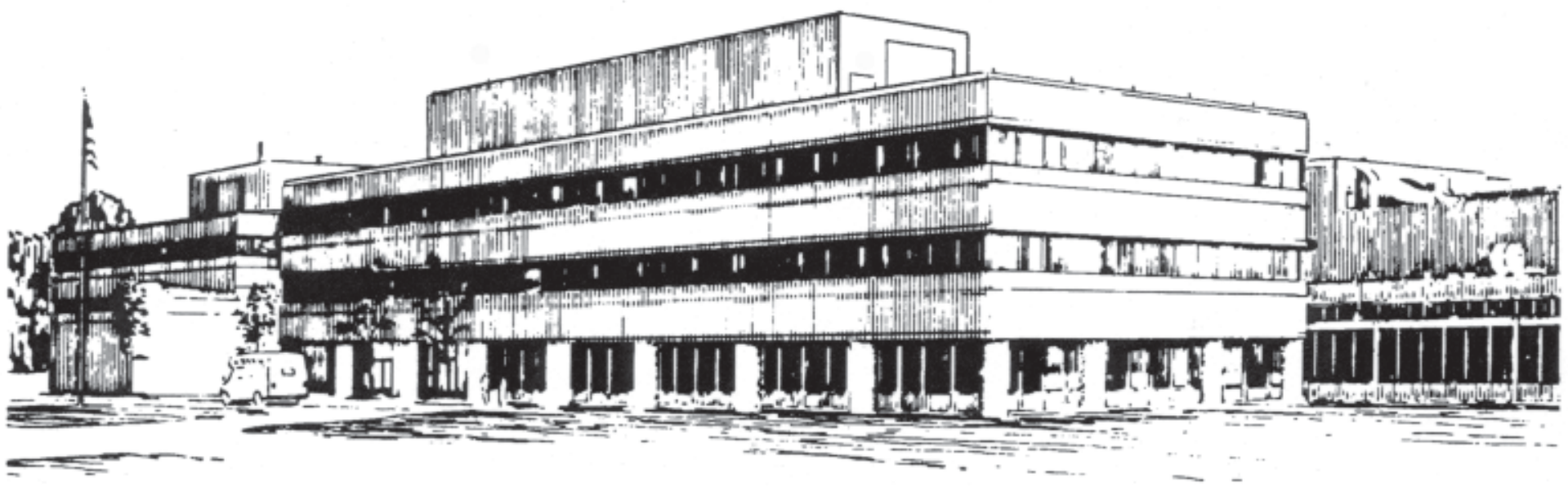

PRINCETON PLASMA PHYSICS LABORATORY PRINCETON UNIVERSITY, PRINCETON, NEW JERSEY 


\section{PPPL Reports Disclaimer}

This report was prepared as an account of work sponsored by an agency of the United States Government. Neither the United States Government nor any agency thereof, nor any of their employees, makes any warranty, express or implied, or assumes any legal liability or responsibility for the accuracy, completeness, or usefulness of any information, apparatus, product, or process disclosed, or represents that its use would not infringe privately owned rights. Reference herein to any specific commercial product, process, or service by trade name, trademark, manufacturer, or otherwise, does not necessarily constitute or imply its endorsement, recommendation, or favoring by the United States Government or any agency thereof. The views and opinions of authors expressed herein do not necessarily state or reflect those of the United States Government or any agency thereof.

\section{Availability}

This report is posted on the U.S. Department of Energy's Princeton Plasma Physics Laboratory Publications and Reports web site in Fiscal Year 2003. The home page for PPPL Reports and Publications is: http://www.pppl.gov/pub_report/

DOE and DOE Contractors can obtain copies of this report from:

U.S. Department of Energy

Office of Scientific and Technical Information

DOE Technical Information Services (DTIS)

P.O. Box 62

Oak Ridge, TN 37831

Telephone: (865) 576-8401

Fax: (865) 576-5728

Email: reports@adonis.osti.gov

This report is available to the general public from:

National Technical Information Service

U.S. Department of Commerce

5285 Port Royal Road

Springfield, VA 22161

Telephone: $1-800-553-6847$ or

(703) $605-6000$

Fax: (703) 321-8547

Internet: http://www.ntis.gov/ordering.htm 


\title{
Ion acceleration in plasmas emerging from a helicon-heated magnetic-mirror device
}

S.A. Cohen, N.S. Siefert, and S. Stange, Princeton Plasma Physics Laboratory, Princeton, NJ 08543

R.F. Boivin* and E.E. Scime, West Virginia University, Morgantown, WV 26506

F.M. Levinton, Nova Photonics, Inc., Princeton, NJ 08540

\begin{abstract}
Using laser-induced fluorescence, measurements have been made of metastable argon-ion, $\operatorname{Ar}^{+*}\left(3 d^{4} F_{7 / 2}\right)$, velocity distributions on the major axis of an axisymmetric magnetic-mirror device whose plasma is sustained by helicon wave absorption. Within the mirror, these ions have sub-eV temperature and, at most, a subthermal axial drift. In the region outside the mirror coils, conditions are found where these ions have a field-parallel velocity above the acoustic speed, to an axial energy of $\sim 30 \mathrm{eV}$, while the field-parallel ion temperature remains low. The supersonic $\operatorname{Ar}^{+*}\left(3 d^{4} F_{7 / 2}\right)$ are accelerated to one-third of their final energy within a short region in the plasma column, $\leq 1 \mathrm{~cm}$, and continue to accelerate over the next $5 \mathrm{~cm}$. Neutralgas density strongly affects the supersonic $\operatorname{Ar}^{+*}\left(3 d^{4} F_{7 / 2}\right)$ density.
\end{abstract}

\section{Introduction}

Helicon wave ${ }^{1}$ physics is important in diverse areas ranging from planetary plasmas to particle accelerators to materials-processing applications. The numerous applications, and the desire to understand the basic physics of helicon waves and the plasmas formed by them, have motivated extensive studies of helicon-wave propagation and absorption, with emphasis on the resulting electron energy distribution, (EED). ${ }^{2}$ Laboratory helicon experiments typically produce plasmas of density $10^{10}-10^{13} \mathrm{~cm}^{-3}$ with electron temperatures in the range $3-10 \mathrm{eV}^{3}$ and ion temperatures below 0.5 eV. ${ }^{4}$ Tenuous electron beams with energies up to $\sim 100 \mathrm{eV}$ have also been documented. ${ }^{5}$ This paper reports on energetic ion beams formed during the expansion of a helicon plasma from a magnetic nozzle. Mechanisms responsible for an observed low-energy metastable-Ar-ion component in the expansion region are also discussed.

These results are especially relevant to a recently proposed application of helicon plasmas: generating intense, collimated, and sustained supersonic plasma streams for spacecraft propulsion, particularly to remote planets. ${ }^{6}$ For propulsion, ion momentum is the relevant parameter. Previous helicon plasma experiments have shown, at most, ion flow at the ion thermal speed. ${ }^{7-9}$ Consequently, some researchers consider auxiliary heating necessary to generate the higher ion energies (exhaust velocities) required for improved propulsion, ${ }^{10}$ though the propulsion community has long been aware that ions in a plasma can be accelerated by electric fields created by ambipolar flow. ${ }^{11}$

Since the 1960s it has been known that inhomogeneous magnetic fields, like the magneticmirror geometry, may create a directed supersonic plasma stream flowing out of the region of high field strength. An early study of Q-machine cesium plasmas expanding along a magnetic field of decreasing strength showed acceleration to $1.7 \times 10^{5} \mathrm{~cm} / \mathrm{s}$ over a distance of $50 \mathrm{~cm} .{ }^{12}$ These results were interpreted in terms of a "magnetic Laval nozzle" with isothermal particles. Some subsequent Q-machines did not show supersonic flow, even with inhomogeneous magnetic fields, ${ }^{13}$ while magnetized and unmagnetized Laval-nozzle-type plasma experiments in other configurations did. ${ }^{14,15}$ Hall-effect magnetic nozzles have accelerated helium and hydrogen plasmas to velocities of order $10^{7} \mathrm{~cm} / \mathrm{s}$ in a distance of $20 \mathrm{~cm} \cdot{ }^{16}$ In marked contrast, a Q-machine experiment in a double-plasma-device (DPD) configuration, showed a thin double-layer electrostatic structure with 
lower potential at the mirror point, implying rapid ion acceleration into the region of higher field strength. ${ }^{17}$

Experiments on double layers in magnetized DPDs measured ion and electron energy distributions with gridded energy analyzers and Langmuir probes inserted into the plasma and found, even in the absence of a magnetic-field gradient, energetic ion beams attributed to spatially inhomogeneous and anisotropic EEDs. ${ }^{18,19}$ The EED may be quite different in the denser helicon plasmas than those in DPDs or Q-machines. Hence, it is possible that double layers or supersonic-Lavalnozzle conditions would not form in magnetic-mirror helicon plasmas or, if they do form, may be in a region not optimal for generating directed plasma exhaust streams. The present paper is the first to report on spatially resolved non-invasive measurements of supersonic ion flows created in helicon-wave-heated plasmas without the use of accelerator or auxiliary-heating techniques. These studies include the parameter range $\omega_{p e} / \omega_{c e} \geq 1$ in which double layers are uncommon ${ }^{20}$ and also explore ranges of magnetic-field gradients and mirror ratios which might inhibit flow.

Our experiments rely on the laser-induced-fluorescence (LIF) technique for non-invasive and precise measurements of ion velocity distributions. ${ }^{21}$ To measure velocity distributions by LIF, the laser frequency is tuned over a range corresponding to the Doppler-shifted line resonance of ions or atoms moving with velocity v. A novel, inexpensive, low-power $(10 \mathrm{~mW})$, tunable, solid-state diode-laser system is used. ${ }^{22}$

\section{Experimental setup}

The experiments were performed in the Magnetic-Nozzle-Experimental facility (MNX), Fig. 1. Steady-state argon plasma is formed by a helicon antenna of the double-saddle type placed around a 4-cm-ID, 30-cm-long Pyrex tube. The $\sim 4-\mathrm{cm}$-diameter plasma flows along the magnetic field formed by a Helmholtz-coil pair placed coaxially around the 45-cm-long, 20-cm-ID, stainless-steel main chamber. The plasma exits the main chamber through the coaxial 2-cm-ID, 3-cm-long nozzle coil used to control the field gradient and mirror ratio. Fig. 1c shows the axial field strength near the nozzle at a Helmholtz coil current of $50 \mathrm{~A}$ and nozzle current of $400 \mathrm{~A}$, typical of experimental conditions in this paper.

Exiting the nozzle coil, the plasma enters a 10-cm-ID, 100-cm-long Pyrex tube termed the expansion region (ER). The ER has 15 internal 4-cm-ID coaxial copper rings, of which 8 may be electrically biased. Additionally there are 3 metal discs, labeled M1, M2, and M3 in Fig. 1a, which may be electrically biased. (In the experiments described here, M1, M2 and M3 were left electrically floating. Biasing M1 had little effect on the plasmas or LIF results; connecting M2 or M3 to ground required more RF power input to sustain the plasma but had little effect on the LIF results.) The disc M2 has a 1-cm-ID hole which limits both the plasma and neutral gas flow into the ER. In the absense of plasma and in conjunction with the ER pump, (P2, see Fig. 1a), the low conductance of M2 maintains up to a $\times 10$ lower pressure in the ER compared with the main chamber. Closing valves V2 and V3 allows controlled increase of the pressure in the ER. Pressures are measured in the main chamber and the ER by two capacitance manometers with accuracies of \pm 0.1 and $\pm 0.001 \mathrm{mT}$, respectively.

At low Helmholtz fields, $B_{H}=300-1200 \mathrm{G}$, MNX stably operates in the helicon mode over a wide range of main-chamber pressures, from 0.4 to above $30 \mathrm{mT}$, at RF powers from 200 to over 2000 W. For reasons that will be clear later, the results described herein were obtained near the lowest main-chamber pressure. The helicon antenna was operated at $26.75 \mathrm{MHz}$. Negligible RF 


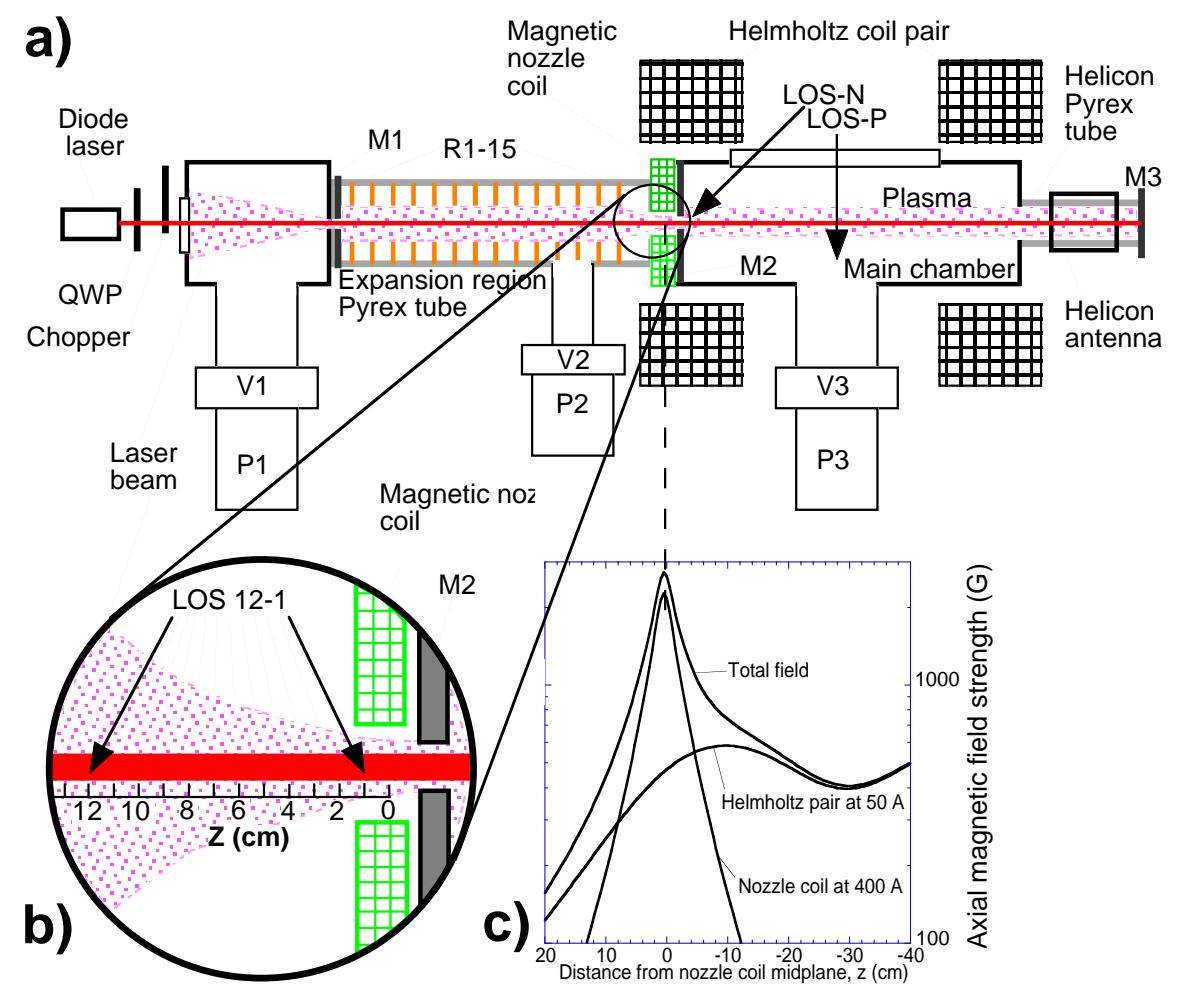

Figure 1: Schematic of the Magnetic Nozzle Experiment (MNX). a) Argon plasma is formed by absorption of helicon waves launched from a double-saddle antenna. The plasma flows through the main chamber along magnetic field fines created by a set of Helmholtz coils. The plasma then flows through metal aperture M2 and the nozzle coil into the expansion region (ER). The beam of a diode laser is directed along the MNX axis, allowing LIF measurements throughout MNX. b) Scanning mechanism for the LIF collection optics allows 12 lines-of-sight (LOS) intercepting axial points in the ER near the nozzle. c) Axial magnetic field strength near the nozzle coil.

is detected in the ER, because of efficient helicon absorption and because of M2. Langmuir probe spatial scans along LOS-P (see Fig. 1a) showed that the plasmas in the main chamber achieved ion densities up to $\sim 2 \times 10^{13} \mathrm{~cm}^{-3}$ and electron temperatures of 4-9 eV. Probe characteristics showed the signature ${ }^{5}$ of 50-100 eV electron beams in both the main and expansion chambers. (Probe measurements in the expansion chamber were along LOS-12, see Fig. 1b.) The floating potentials of the copper rings in the ER were typically -40 to -120 volts, further evidence for electron beams. (Biasing the rings to \pm 40 volts had negligible effect on the LIF results, all of which pertain to plasma on the MNX axis.)

To permit measurement of the field-parallel $\mathrm{Ar}^{+*}$ velocity distribution in MNX, the ellipticalcross-section $(5 \mathrm{~mm} \times 1 \mathrm{~mm})$ tuneable diode-laser beam is directed along the MNX magnetic axis. Optics to collect the fluorescence emission are located on both the main chamber (two lines-of-sight, LOS, Fig. 1a) and in the expansion chamber (typically 12 LOS, Fig. 1b). One main-chamber LOS (LOS-P) collects from a segment of the plasma in the center of the chamber; the other, LOS$\mathrm{N}$, collects photons from the plasma near M2 and extending $1.2 \mathrm{~cm}$ back into the main chamber. Scanning optics on the ER allow LOS which intercept the laser beam from 1-cm from the nozzle-coil midplane to $12 \mathrm{~cm}$ from its midplane, as well as beyond, see $z$-axis in Fig. $1 \mathrm{~b}$. 
The 1.5-MHz bandwidth Sacher LaserTechnik diode laser may be coarse tuned in wavelength from 662 to $674 \mathrm{~nm}$, allowing excitation of the $668.614 \mathrm{~nm}$ (zero-field, vacuum) transition $3 d^{4} F_{7 / 2}-$ $4 p^{4} D_{5 / 2}$ of $\mathrm{Ar}^{+*} .^{22}$ Finer tuning, over a maximum $0.4 \mathrm{~nm}$ wavelength range, is accomplished manually or automatically, by changing the voltage on the laser's internal piezoelectric crystal. A photomultiplier with 1-nm transmission filter centered at $442.7 \mathrm{~nm}$ (vacuum) collects emission from the $442.7 \mathrm{~nm}$ fluorescence transition, $4 p^{4} D_{5 / 2}-4 s^{4} P_{3 / 2}$, with Einstein coefficient $A=9.856 \times 10^{7} s^{-1} .{ }^{23}$ The Zeeman effect from the Helmholtz and nozzle coil fields separates the $668.614 \mathrm{~nm}$ transition into six $+\sigma$, six $-\sigma$, and six $\pi$ components. To reduce the complexity of the LIF spectrum and increase signal/noise $(\mathrm{S} / \mathrm{N})$, the linearly polarized laser beam may be passed through a quarterwave plate (QWP), oriented to convert the beam into either right- or left-circularly polarized (RCP, LCP) light for exciting either the + or $-\sigma$ transitions. The (circularly polarized) laser beam is then modulated at $\sim 4 \mathrm{kHz}$ by a mechanical chopper, for lock-in detection of the $442.7 \mathrm{~nm}$ fluorescence. This LIF system operates well within the unsaturated mode wherein the LIF signal is proportional to the laser intensity and the $3 d^{4} F_{7 / 2}$ metastable population in the detection volume, i.e., observation volume $\cap$ irradiation volume, that is in resonance with the laser. A single wavelength scan is usually limited to a mode-hop-free region of $0.021 \mathrm{~nm}(14 \mathrm{GHz})$ and is performed in $\sim 60$ seconds; 1000 data points are recorded. A set of observations of a single plasma condition typically includes scans over several adjacent wavelength regions in the range 668.580 to $668.660 \mathrm{~nm}$, both with and without the QWP inserted. Using orthogonal QWP orientations allows the Zeeman splitting to be measured and the (unshifted) line center to be identified. The laser wavelength is measured with two Burleigh wavemeters, one accurate to $\pm 0.001 \mathrm{~nm}$, the other to $\pm 0.0001 \mathrm{~nm}$.

\section{Results and discussion}

Figure 2 shows a set of four LIF amplitude vs. wavelength (frequency) scans at LOS-1 in the ER. Two were over the range 668.603-668.624 nm and two for 668.625-668.646 nm, $\pm 0.001 \mathrm{~nm}$. (0 $\mathrm{GHz}$ on the abscissa corresponds to $668.614 \mathrm{~nm}$, i.e., the unshifted line.) For each pair in each wavelength range, the QWP was used, first to create a RCP beam, then a LCP beam. The experimental conditions were: absorbed helicon power $\mathrm{P}_{f}=830 \mathrm{~W} ; \mathrm{LOS} \mathrm{z}=1 \mathrm{~cm} ; B_{H}=600$ G; $B_{N}=625 \mathrm{G}$; ER pressure $p_{E R} \sim 0.15$ mTorr; and main chamber pressure $\mathrm{p}_{M} \sim 0.6$ mTorr. Langmuir probes showed the on-axis electron density and temperature to be $6 \pm 2 \times 10^{12} \mathrm{~cm}^{-3}$ and $7.0 \pm 0.2 \mathrm{eV}$ in the main chamber and $2.6 \pm 0.4 \times 10^{10} \mathrm{~cm}^{-3}$ and $9.3 \pm 0.3 \mathrm{eV}$ at $z=10 \mathrm{~cm}$ in the ER. Note that $\beta \equiv 8 \pi \sum_{j=i, e} n_{j} k T_{j} / B^{2} \ll 1$.

Each LIF scan shows a peak. The two prominent peaks separated by $\pm 1.4 \mathrm{GHz}$ from $0 \mathrm{GHz}$ are the + and $-\sigma$ Zeeman components of cool $\left(T_{i, \|} \sim 0.2 \mathrm{eV}\right) \operatorname{Ar}^{+*}\left(3 d^{4} F_{7 / 2}\right)$. The 12 separate Zeeman $\sigma$ components, whose amplitudes and spacing are shown in the figure, are not resolved due to Doppler broadening and magnetic-field variation over the spatial extent of the detection volume. These two peaks centered about the unshifted $3 d^{4} F_{7 / 2}-4 p^{4} D_{5 / 2}$ transition are termed the Low Energy Peaks (LEP).

Two additional peaks are seen in the redder part of the spectrum, at Doppler shifts of -8 to $-20 \mathrm{GHz}$. The center of this pair of peaks is at $-13.5 \mathrm{GHz}$, corresponding to an axial energy of $17 \pm 2 \mathrm{eV}$. These peaks are referred to as the High Energy Peaks (HEP). The HEP show a tail on their low energy side.

The first question is what are the sources for the LEP and the HEP at this position in the ER. Possible sources for $\operatorname{Ar}^{+*}\left(3 d^{4} F_{7 / 2}\right)$ in this detection volume are: 1) combined ionization/excitation 


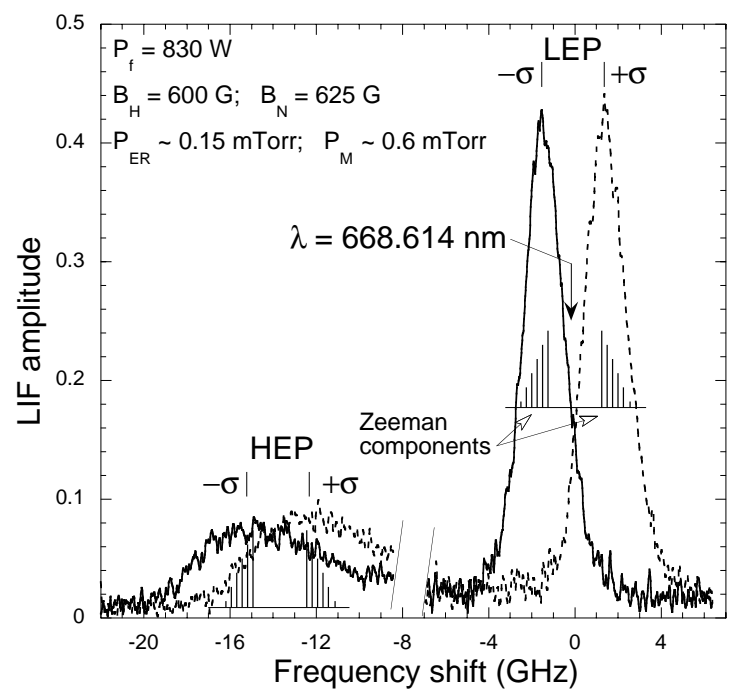

Figure 2: Laser-induced fluorescence amplitude vs. frequency shift at $z=1 \mathrm{~cm}$ in the ER. $0 \mathrm{GHz}$ corresponds to the unshifted quartet $3 d^{4} F_{7 / 2}-4 p^{4} D_{5 / 2}$ wavelength. The Zeeman effect splits the quartet into + and - $\sigma$ components. Peaks near $0 \mathrm{GHz}$ are termed the Low Energy Peaks (LEP). Peaks with frequency shifts greater than $\sim 6 \mathrm{GHz}$ are termed High Energy Peaks (HEP).

of cold Ar neutrals by the plasma electrons (both thermal and beam-like); 2) excitation of $\mathrm{Ar}^{+}$by the plasma electrons; 3$)$ cascades from higher $\mathrm{Ar}^{+}$excitation levels; and 4) flow of $\mathrm{Ar}^{+*}\left(3 d^{4} F_{7 / 2}\right)$ along or across the magnetic field. Ions of the latter type may gain energy if there is a potential difference between locations. Deexcitation of the metastables - also called quenching — by collisions with neutrals ${ }^{24}$ may occur. Two-step processes may also contribute to the $\operatorname{Ar}^{+*}\left(3 d^{4} F_{7 / 2}\right)$ population but are generally less important than the processes named above.

We shall now present evidence that the HEP is due to $\operatorname{Ar}^{+*}\left(3 d^{4} F_{7 / 2}\right)$ formed within a few mm of the M2/nozzle in the main chamber and accelerated through the nozzle and that the LEP is due to ionization of background neutral gas by the plasma electrons in the ER.

If the plasma in the expansion region were due solely to plasma bound to field lines and directly flowing out the nozzle, then the LEP and HEP amplitudes would decrease with increasing $z$ because of field expansion. As shown in Fig. 3, the HEP amplitude -actually the area under the HEP peak from $\mathrm{E}=6$ to $40 \mathrm{eV}$ - does decrease with distance but the LEP amplitude - the height of LEP peak - grows with increasing distance from the nozzle. This is the first piece of evidence that the the LEP is not simply plasma directly flowing from the nozzle. (The operating conditions were similar to those of Fig. 2: absorbed helicon power $P_{f}=900 \mathrm{~W} ; B_{H}=575 \mathrm{G} ; B_{N}=1400 \mathrm{G} ; p_{E R}=0.135$ mTorr; and $\mathrm{p}_{M}=0.5$ mTorr.) The energy of the HEP peak, $E_{H E P}$, also shown in Fig. 3, increases with increasing $z$. Close to the nozzle the energy is about $11 \mathrm{eV}$; at the farthest position with adequate $\mathrm{S} / \mathrm{N}$ to measure $E_{H E P}$ accurately, $z \sim 5.5 \mathrm{~cm}, E_{H E P}$ reaches $30 \mathrm{eV}$, corresponding to a Mach number of about 2 for $T_{e}=7 \mathrm{eV}$, the bulk electron temperature in the main chamber.

In the Laval-nozzle picture, ions would attain the sound speed $\sqrt{T_{e} / m_{i}}-$ corresponding to an axial energy of $3.5 \mathrm{eV}$ - at the nozzle throat and a maximum energy at infinite expansion of $\gamma T_{e} /(\gamma-1)=17.5 \mathrm{eV}$ for a polytropic coefficient $\gamma=5 / 3$. Note that at $z=5.5 \mathrm{~cm}$, the magnetic expansion is only a factor of 3 from the nozzle throat. The data clearly show greater ion acceleration 
near the nozzle than the simple Laval-nozzle model predicts.

The continuing increase in ion energy with $z$ is in qualitative agreement with the prediction of Ref. 11, based on an ambipolar-flow, thermal-conduction model. Quantitative agreement with Ref. 11 for the ratio of final ion energy to upstream electron temperature can be achieved by selecting the location of transition to supersonic flow to be at $z \sim 1 \mathrm{~cm}$. The MNX data do not show the axial decrease of electron temperature predicted by this thermal-conduction model.

Spatial scans for $400 \leq P_{H} \leq 1200 \mathrm{~W}$ and $300 \leq B_{H} \leq 1200 \mathrm{G}$ showed similar results, that the HEP amplitude falls and $E_{H E P}$ rises with $z$. The parallel ion temperature, estimated from the LIF full width at half height, also rises with $z$. LEP spatial scans show a more complex behavior whose discussion is deferred to a later paper.

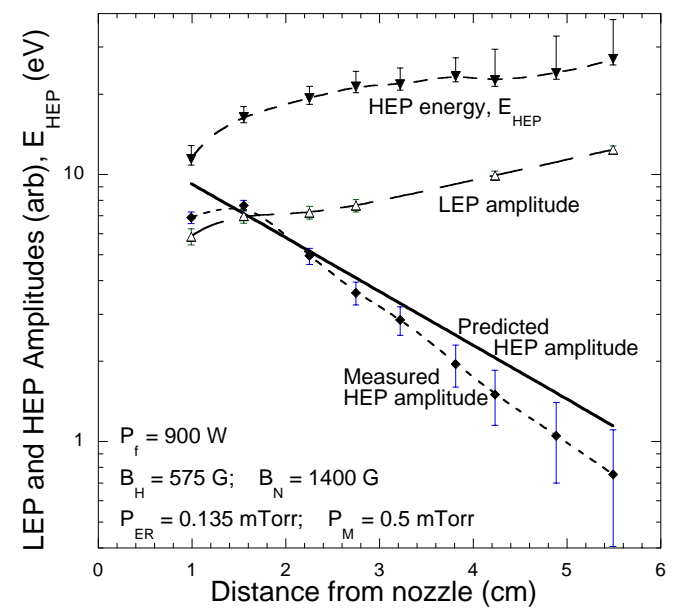

Figure 3: LEP and HEP amplitudes and HEP energy versus distance from the nozzle midplane. The model used to predict the HEP amplitude is based on plasma tied to field lines, the continuity equation, and metastable quenching by collisions with neutrals.

LIF measurements in the main chamber, along LOS-P and LOS-N, only showed a clear LEP, never a HEP. The ion temperatures were in the ranges $0.04-0.12 \mathrm{eV}$ for $T_{i, \|}$ and $0.05-0.5 \mathrm{eV}$ for $T_{i, \perp}$. The axial drift of the $A r^{+*}\left(3 d^{4} F_{7 / 2}\right)$ in the main chamber was small, comparable or less than the ion thermal speed. We conclude that the HEP is due to ions accelerated during their passage through the nozzle. Later we shall note why the bulk ions in the main chamber do not necessarily have the same negligible drift velocity as the metastable ions.

To understand the behavior of the HEP amplitude $\left(\mathrm{A}_{H E P}\right)$ vs. $z$, it is necessary to include (at least) effects of magnetic field expansion, ion-energy gain with $z$, and $\operatorname{Ar}^{+*}\left(3 d^{4} F_{7 / 2}\right)$ collisions with the background neutral gas. To address these, ER gas pressure, $\mathrm{p}_{E R}$, and nozzle-coil field strength, $B_{N}$, were changed and characteristics of HEP and LEP in the ER were measured. For the same Helmholtz field as in Fig. 3 but only $440 \mathrm{~W}$ of helicon power, Fig. 4 shows that the LEP brightness at $z=2 \mathrm{~cm}$ grows linearly with $p_{E R}$ in the range 0.2 to 2 mTorr (at constant $\left.p_{M}\right)$, consistent with a picture that the low energy $\operatorname{Ar}^{+*}\left(3 d^{4} F_{7 / 2}\right)$ are created by ionization of the neutrals in the ER. In contrast, the HEP decreases exponentially with pressure, with a $1 / e$ characteristic fall-off at pressure increments of $\delta p_{e}=0.274$ mTorr. Assuming the neutrals are in thermal equilibrium with the wall because of the low pressure, $\delta p_{e}$ yields a collisional quenching- 
cross-section of the metastable state $Q_{m}=515 \pm 100 \AA^{2}$, about a factor of 2 larger than that measured for the metastable state $\operatorname{Ar}^{+*}\left(3 d^{2} G_{9 / 2}\right)$ at $0.1-5 \mathrm{eV} \cdot{ }^{24}$ (Perhaps it is more proper to call our measurement an effective-quenching-cross-section because collisions with neutrals might also cause radial transport, or collisions with electrons might cause quenching, both resulting in an attendant decrease in LIF signal.) We then calculate the predicted HEP amplitude as a function of $z$ assuming the $\operatorname{Ar}^{+*}\left(3 d^{4} F_{7 / 2}\right)$ remains on field lines and including $Q_{m}$ and the measured $z$ dependence of $\mathrm{E}_{H E P}$, i.e., $\int_{6 e V}^{40 \mathrm{eV}} A_{H E P} d E \propto B(z) \exp \left[-z n_{n} Q_{m}\right] / E_{H E P}^{0.5}$, where the $34 \mathrm{eV}$ range-ofintegration for energy includes the broadening of the HEP with distance, $n_{n}$ is the neutral density, and $\mathrm{B}(\mathrm{z})$ is the field strength at a point $z$. As shown in Fig. 3, the predicted result decreases less rapidly with distance than does the measured LIF amplitude. Possible causes for the discrepancy are radial transport ${ }^{25}$ though not that caused by collisions with neutrals, and quenching by electron impact. (Low- $\beta$, axisymmetric plasmas are expected to follow field lines.) The observed continuing ion acceleration with increasing $z$ is qualitatively as predicted by several groups. ${ }^{11,26}$

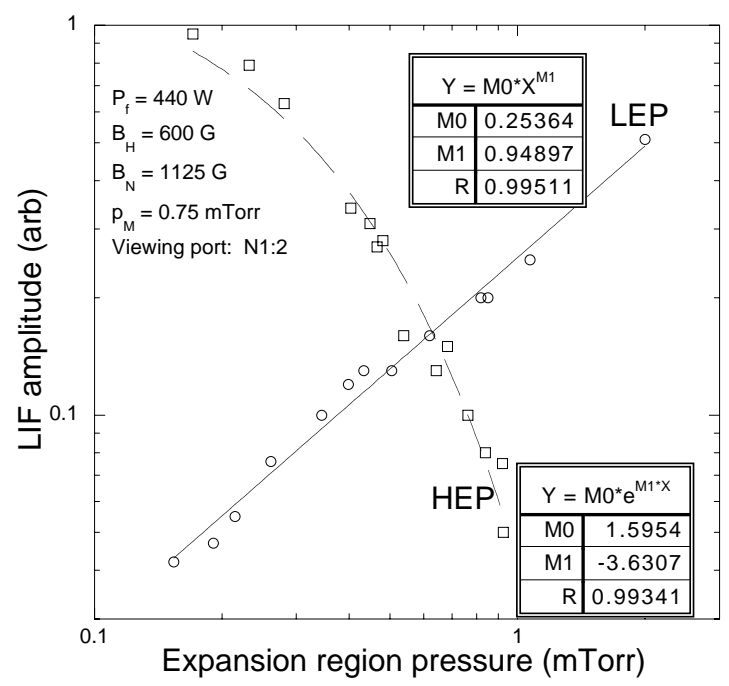

Figure 4: Dependence of HEP and LEP amplitudes on expansion region pressure at $z=2 \mathrm{~cm}$ in the ER.

The final result reported in this paper is the effect of the nozzle field on the metastable ions in the ER. Figure 5 shows the behavior of HEP and LEP vs. the nozzle field strength. The experimental conditions are: $B_{H}=1200 \mathrm{G}$; absorbed helicon power $\mathrm{P}_{f}=450 \mathrm{~W}$; LOS z $=2$ $\mathrm{cm}$; low ER pressure $0.15 \leq p_{E R} \leq 0.2 \mathrm{mTorr}$; high ER pressure $0.72 \leq p_{E R} \leq 0.85 \mathrm{mTorr}$; and main chamber pressure $\mathrm{p}_{M} \sim 0.8$ mTorr. Ranges of $p_{E R}$ are given because $p_{E R}$ increases when the nozzle-coil current is increased, apparently channeling more plasma into the ER. Though increased magnetic-mirror ratio at the nozzle coil might be expected to reduce the flux through the nozzle coil, this is not the case. We note that the 4-cm diameter of the plasma column in the main chamber is wide compared to the 1-cm diameter M2 orifice and the ions in the main chamber are collisional: both these facts act against decreased ion transmission at higher mirror ratios. The amplitudes of the HEP at both low and high pressure increase approximately proportional to the magnetic flux through the nozzle coil. The LEP at low pressure also increases linearly with nozzle field strength. The percentage increase in LEP amplitude can be explained by two effects: the increase in $p_{E R}$ due to the nozzle coil and, more importantly, the increase in plasma density (both thermal electron and 
beam electron densities) in the ER. Discussion of the LEP behavior at high pressure is deferred to a later paper.

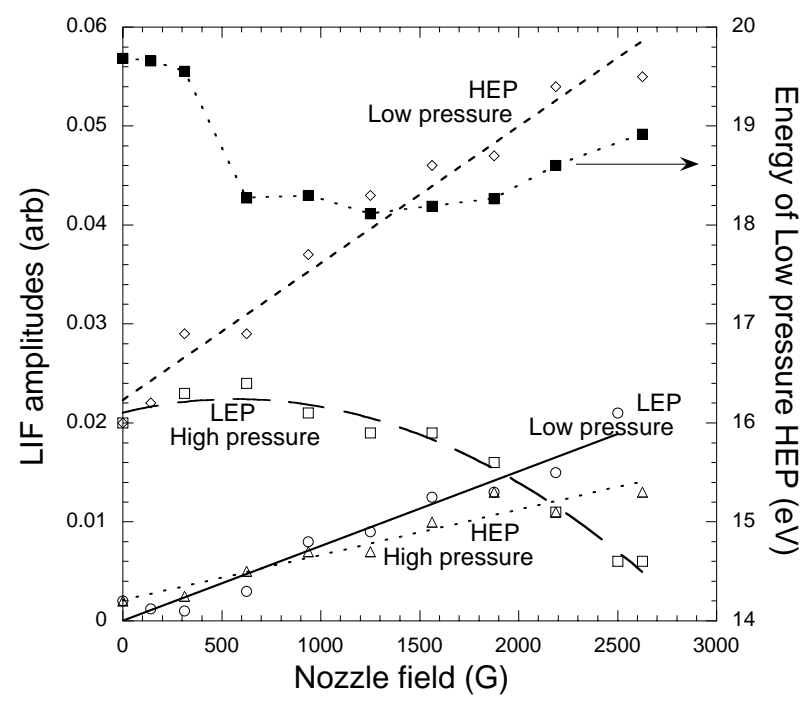

Figure 5: Effect of nozzle magnetic field strength on the LEP and HEP amplitudes for two ER pressures. The energy of the HEP at low $p_{E R}$ is also shown. The absolute value of energy is uncertain to $\pm 2 \mathrm{eV}$; the relative value to $\pm 0.4 \mathrm{eV}$. The plasma conditions were: $P_{f}=450 \mathrm{~W} ; B_{H}=1200 \mathrm{G}$; and LOS $z=2 \mathrm{~cm}$.

For the low-pressure nozzle-field scan, the HEP S/N was sufficient to allow precise measurements of energy. After an initial drop of $6 \%, E_{H E P}$ stays nearly constant as the nozzle field is increased a factor of 5. At zero nozzle current, the mirror point is at $z=-10 \mathrm{~cm}$ (see Fig. 1c), while at higher currents it moves to $z=0 \mathrm{~cm}$. If the ion acceleration to above $11 \mathrm{eV}$ occurred at the mirror point, then we would expect appreciable quenching at zero nozzle current, because of the 0.8 mTorr pressure in the main chamber and the $12-\mathrm{cm}$ distance to the detection volume. Thus this data localizes the region of HEP energy gain closer to M2 than to the position of the mirror point.

From the low value of ion temperature in the main chamber and the lack of increased ion energy at higher nozzle fields, it is seen that both the ion acceleration in the nozzle and their continuing parallel acceleration in the $5 \mathrm{~cm}$ beyond the nozzle are not due to ion pressure or magnetic-moment conservation, but to an axial electric field. The more abundant thermal electrons seem a more likely source for the electric field than beam electrons, based on the maximimum observed $E_{H E P} \approx 4 T_{e}$. The short length of the $\mathrm{M} 2 /$ nozzle ion-acceleration region, $\leq 1 \mathrm{~cm}$, leads us to suggest that a double layer exists there. The boundary between the main-chamber plasma and the expansionregion plasma is established by metal plate, M2, rather than by the throat of the nozzle magnetic field. The near-nozzle/M2 electric field should cause cold electrons created in the ER to flow back into the main chamber. We estimate that drag created by these electrons has little effect on the HEP ions.

For the experiments reported here, the helicon was typically operated at $p_{M} \sim 0.5$ mTorr and a resultant neutral-induced collisional quenching mean-free-path for the $3 d^{4} F_{7 / 2}$ metastable state of $\lambda_{n, *} \leq 1 \mathrm{~cm}$. Operation of helicon discharges at higher pressures, above 2 mTorr, results in $\lambda_{n, *} \leq$ few mm. At these higher pressures, LIF may be less applicable to the diagnosis of 
certain phenomena, e.g., acceleration in a nozzle or perhaps ion heating, which might take place over distances larger than a few mm. An axial electric field might exist in the main chamber, accelerating ions towards the orifice in M2. Metastables, however, are quenched by collisions with neutrals in the main chamber and never show this early energy gain. Bulk ions, predominantly in the ground state, may have this feature.

The highest argon ion energy observed in the expansion region, $E_{H E P}=30 \mathrm{eV}$, corresponds to a specific impulse, $I_{s p}\left(\equiv \mathbf{v} / g\right.$, where $\left.g=9.8 \mathrm{~m} / \mathrm{s}^{2}\right)$ of $1100 \mathrm{~s}$, about a factor of 3 lower than the minimum considered desirable for remote-planet missions. Methods to increase $I_{s p}$ might include the aforementioned auxiliary heating or use of a lower mass propellant. Once the requisite $I_{s p}$ threshold is crossed, additional technical issues must be addressed, e.g., energy efficiency, propellant utilization efficiency, and engine reliability and lifetime, for helicon-heated plasmas in a magneticnozzle geometry to be suitable for the proposed ambitious use.

\section{Summary}

We have found operating conditions for axisymmetric magnetic-mirror-geometry argon helicon discharges which generate supersonic metastable-ion flow though an orifice on the magnetic axis. The argon ion energy increases sharply at the orifice and then continues to increase to $\sim 4 \times$ the electron temperature in the main discharge. The parallel ion temperature remains low, $\leq 2 \mathrm{eV}$. For a small orifice/plasma-diameter ratio, the supersonic metastable-ion beam intensity grows with magnetic flux through the orifice. A population of low energy, $\leq 0.5 \mathrm{eV}$, slowly flowing metastable ions is found in the expansion region. If the neutral pressure is allowed to rise above $10^{-4}$ Torr, the low-energy metastable-ion population grows considerably while the supersonic metastable ion density falls.

\section{Acknowledgements}

We are grateful to B. Berlinger, V. Corso, R. Feder, R. Gondhalekar, A. Keesee, G. Lemunyan, and K. Stokke for excellent technical assitance and to T.K. Chu for his comments on the manuscript. This work was supported, in part, by U.S. Department of Energy Contract No. DE-AC02-76-CHO3073 . 


\section{References}

[*] present address: 211 Allison Laboratory, Department of Physics, Auburn University, AL 36849.

[1] R.W. Boswell, Phys. Lett. 33A, 457 (1970).

[2] F.F. Chen, Plasma Physics and Controlled Fusion 33, 339 (1991).

[3] For reviews see R.W. Boswell and F.F. Chen, IEEE Transactions on Plasma Science 25, 1229 (1997) and F.F. Chen and R.W. Boswell, IEEE Transactions on Plasma Science 25, 1245 (1997).

[4] E.E. Scime, P.A. Keiter, M.W. Zintl, et al., Plasma Sources Sci. and Technol. 7, 186 (1998).

[5] R. T. S. Chen and N. Hershkowitz, Phys. Rev. Lett. 80, 4677 (1998).

[6] F.R. Chang-Diaz, "The VASIMR Rocket", Scientific American, (Nov. 2002).

[7] J.L. Kline, E.E. Scime, P.A. Keiter, et al., Phys. Plasmas 6, 4767 (1999).

[8] J.L. Kline, E.E. Scime, R.F. Boivin, et al., Phys. Rev. Lett. 88 , 195002 (2002).

[9] While this paper was being written, we were informed by F. Chang Diaz of supersonic ion beams found in helium and hydrogen helicon plasmas (private communication, Sept 2002 and J.P. Squire, F.R. Chang Daz, T.W. Glover, et al., Transactions of Fusion Science and Technology, accepted July 2002).

[10] F. Chang-Diaz, submitted Transactions of Fusion Technology (July 2002).

[11] E.L. Walker and G.R. Seikel, "Axisymmetric expansion of a plasma in a magnetic nozzle including thermal conduction," NASA TN D-6154 (February 1971) and references therein.

[12] S.A. Andersen, V.O. Jensen, P. Nielsen, and N. D'Angelo, Phys. Fluids 12, 557 (1969).

[13] M.E. Koepke, M. Zintl, C. Teodorescu, et al., Phys. Plasmas 9, 3225 (2002).

[14] S. Mazouffre, M.G.H. Boogaarts, J.A.M. van der Mullen, and D.C. Schram, Phys. Rev. Lett. 84, 2622 (2000).

[15] P. Engeln, S. Mazouffre, P. Vankan, et al., Plasma Sources Sci. Technol. 11, A100 (2002).

[16] K.F. Schoenberg, R.A. Gerwin, R.W. Moses, et al., Phys. Plasmas 5, 2090 (1998).

[17] R. Hatakeyama, Y. Suzuki, and N. Sato, Phys. Rev. Lett. 50, 1203 (1983).

[18] G. Hairapetian and R.L. Stenzel, Phys. Rev. Lett. 61, 1607 (1988).

[19] G. Hairapetian and R.L. Stenzel, Phys. Rev. Lett. 65, 175 (1990).

[20] P. Coakley, L. Johnson, and N. Hershkowitz, Physics Lett. 70A, 425 (1979).

[21] G.D. Severn, D.A. Edrich, and R. McWilliams, Rev. Sci. Instrum. 69, 10 (1998).

[22] R.F. Boivin, West Virginia University Plasma Physics Lab Report PL-050 (Dec 2001).

[23] R.L. Kurucz and B. Bell, 1995 Atomic Line Data, Kurucz CD-ROM No. 23. Cambridge, MA, Smithsonian Astrophysical Observatory.

[24] F. Skiff, G. Bachet, and F. Doveil, Phys. Plasmas 8, 3139 (2001).

[25] I.D. Kaganovich, V.A. Rozhansky, L.D. Tsendin, and I.Yu. Veselova, Plasma Sources Sci. Technol. 5, 743 (1996).

[26] K. E. Lonngren and N. Hershkowitz, IEEE Transactions on Plasma Science PS-7, 107 (1979). 


\section{External Distribution}

Plasma Research Laboratory, Australian National University, Australia

Professor I.R. Jones, Flinders University, Australia

Professor João Canalle, Instituto de Fisica DEQ/IF - UERJ, Brazil

Mr. Gerson O. Ludwig, Instituto Nacional de Pesquisas, Brazil

Dr. P.H. Sakanaka, Instituto Fisica, Brazil

The Librarian, Culham Laboratory, England

Mrs. S.A. Hutchinson, JET Library, England

Professor M.N. Bussac, Ecole Polytechnique, France

Librarian, Max-Planck-Institut für Plasmaphysik, Germany

Jolan Moldvai, Reports Library, MTA KFKI-ATKI, Hungary

Dr. P. Kaw, Institute for Plasma Research, India

Ms. P.J. Pathak, Librarian, Insitute for Plasma Research, India

Ms. Clelia De Palo, Associazione EURATOM-ENEA, Italy

Dr. G. Grosso, Instituto di Fisica del Plasma, Italy

Librarian, Naka Fusion Research Establishment, JAERI, Japan

Library, Plasma Physics Laboratory, Kyoto University, Japan

Research Information Center, National Institute for Fusion Science, Japan

Dr. O. Mitarai, Kyushu Tokai University, Japan

Dr. Jiangang Li, Institute of Plasma Physics, Chinese Academy of Sciences, People's Republic of China

Professor Yuping Huo, School of Physical Science and Technology, People's Republic of China

Library, Academia Sinica, Institute of Plasma Physics, People's Republic of China

Librarian, Institute of Physics, Chinese Academy of Sciences, People's Republic of China

Dr. S. Mirnov, TRINITI, Troitsk, Russian Federation, Russia

Dr. V.S. Strelkov, Kurchatov Institute, Russian Federation, Russia

Professor Peter Lukac, Katedra Fyziky Plazmy MFF UK, Mlynska dolina F-2, Komenskeho Univerzita, SK-842 15 Bratislava, Slovakia

Dr. G.S. Lee, Korea Basic Science Institute, South Korea

Institute for Plasma Research, University of Maryland, USA

Librarian, Fusion Energy Division, Oak Ridge National Laboratory, USA

Librarian, Institute of Fusion Studies, University of Texas, USA

Librarian, Magnetic Fusion Program, Lawrence Livermore National Laboratory, USA

Library, General Atomics, USA

Plasma Physics Group, Fusion Energy Research Program, University of California at San Diego, USA

Plasma Physics Library, Columbia University, USA

Alkesh Punjabi, Center for Fusion Research and Training, Hampton University, USA

Dr. W.M. Stacey, Fusion Research Center, Georgia Institute of Technology, USA

Dr. John Willis, U.S. Department of Energy, Office of Fusion Energy Sciences, USA

Mr. Paul H. Wright, Indianapolis, Indiana, USA 
The Princeton Plasma Physics Laboratory is operated by Princeton University under contract with the U.S. Department of Energy.

\author{
Information Services \\ Princeton Plasma Physics Laboratory \\ P.O. Box 451 \\ Princeton, NJ 08543
}

Phone: 609-243-2750

Fax: 609-243-2751

e-mail: pppl_info@pppl.gov

Internet Address: http://www.pppl.gov 